\title{
Enteroplastía transversa seriada como alternativa en el tratamiento del síndrome de intestino corto. Caso clínico
}

Departamentos de Cirugía Digestiva y División de Cirugía. ${ }^{2}$ Departamento de Nutrición y Diabetes.

Facultad de Medicina Pontificia Universidad Católica de Chile. anterna de Medicina. Pontificia Universidad Católica de Chile.

Recibido el 26 de junio de 2009, aceptado el 22 de marzo de 2010

Correspondencia a:

Dr. Felipe Bellolio Roth

Unidad de Coloproctología.

Departamento de Cirugía

Digestiva.

División de Cirugía. Facultad de

Medicina Pontificia Universidad

Católica de Chile.

Marcoleta 350 patio interior.

Fono: 3543462

Fax: 6382793

E-mail: fbelloli@med.puc.cl

\author{
FELIPE BELLOLIO R. ${ }^{1}$, JULIETA KLAASSEN L. ${ }^{2}$, DAHIANA PULGAR B. ${ }^{a}$, \\ M $^{\text {a }}$ ELENA MOLINA P. ${ }^{1}$, GEORGE PINEDO M. ${ }^{1}$, ALVARO ZÚÑIGA D. ${ }^{1}$
}

\section{Serial transverse enteroplasty for short bowel syndrome. Case report}

\begin{abstract}
Among patients with short bowel syndrome, surgical small intestine lengthening techniques are employed to increase the absorptive surface. Among these, serial transverse enteroplasty involves transecting the bowel transversally, preserving the blood supply of the small intestine and creating a longer segment of bowel. We report a 51-year-old woman with a short bowel syndrome and multiple hospital admissions for complications. She was subjected to a serial transverse enteroplasty, increasing small intestinal length from 140 to $180 \mathrm{~cm}$. During the postoperative period, she presented intra abdominal blood collections and a septic episode with bacterial endocarditis. One month after the operation, total parenteral nutrition was discontinued and nutritional and fluid balances were achieved using exclusively the oral route. During the ambulatory follow up, the patient continues with exclusive oral feeding and five bowel movements per day.
\end{abstract}

(Rev Med Chile 2010; 138: 478-482).

Key words: Enteroplasty; Parenteral nutrition; Short bowel syndrome.
$\mathrm{E}$ 1 síndrome de intestino corto (SIC) se define como la insuficiencia intestinal ocasionada por la pérdida anatómica o funcional de parte o todo el intestino delgado, lo que genera una deficiente absorción de nutrientes, líquido y oligoelementos $^{1}$. El SIC es particularmente grave en los pacientes con resección de la válvula ileocecal o si el colon ha sido removido.

Los pacientes con SIC requieren para su manejo apoyo con nutrición parenteral total central (NPTC). Diversas series han comunicado complicaciones asociadas a su uso en forma crónica, tales como dificultad para mantener accesos venosos, sepsis por catéter, colestasia y hepatopatía grave $e^{2-4}$. El desarrollo de estas complicaciones califica a los pacientes como candidatos a trasplante intestinal $(\mathrm{TI})^{5,6}$, procedimiento con una tasa de morbilidad elevada y que requiere de inmunosupresión ${ }^{7}$.
Actualmente existen técnicas de elongación intestinal que en algunos pacientes puede evitar el TI. Dentro de ellas destacan la duplicación intestinal descrita por Bianchi ${ }^{8}$ y la enteroplastía transversa seriada o STEP (Serial Transverse EnteroPlasty). Esta última consiste en una serie de secciones transversas parciales sobre el intestino de manera que se crea un canal en zig-zag que reduce el lumen intestinal e incrementa su longitud (Figura 1).

Presentamos el caso clínico de una paciente sometida a STEP.

\section{Caso clínico}

Paciente de sexo femenino, con diagnóstico de inercia colónica. En mayo de 2003, a los 46 años, se le realizó una colectomía total laparoscópica con 


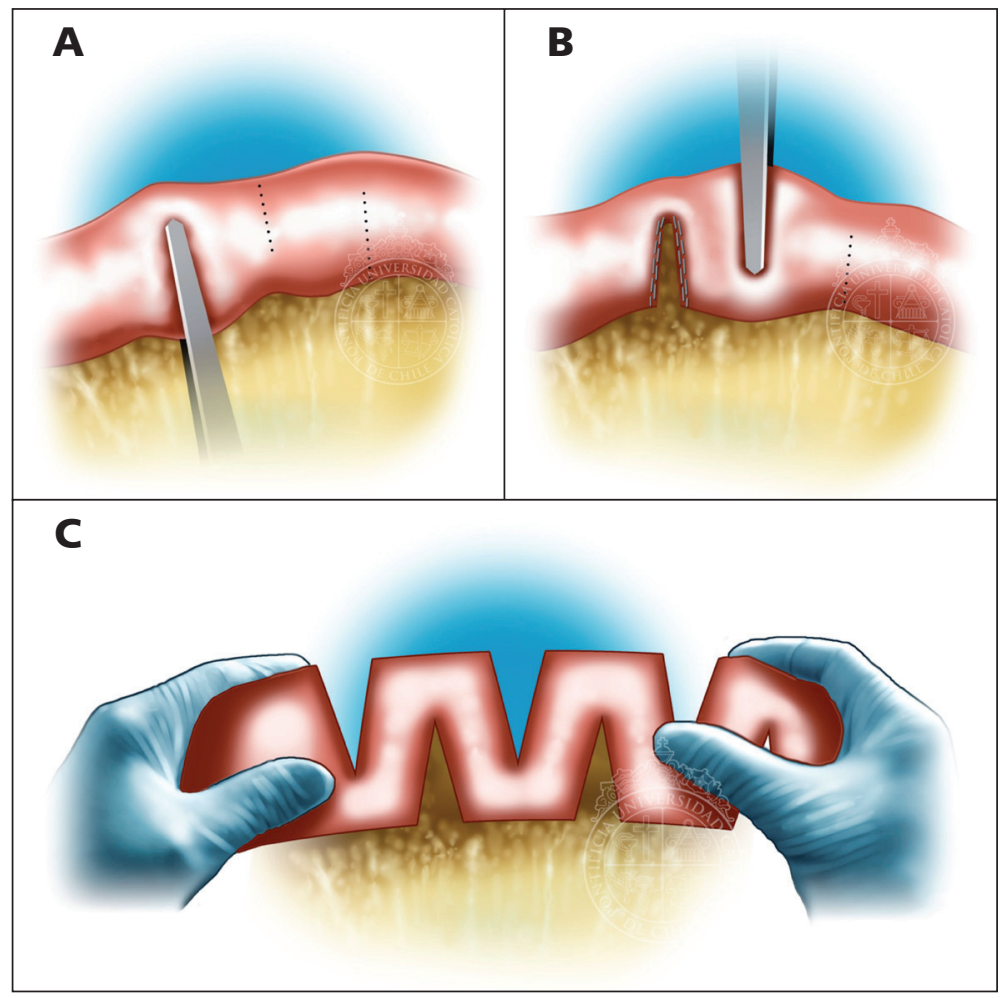

Figura 1. A y B: Enteroplastías transversales. C: Canal con forma de zig-zag. anastomosis ileorrectal sin incidentes. Durante el postoperatorio evolucionó con taquicardia, dolor abdominal e hipotensión. Se practicó una laparotomía exploradora que evidenció necrosis de yeyuno distal e ileon, realizándose resección del intestino desde los $120 \mathrm{~cm}$ distales al Treitz, además de una yeyunostomía terminal y cierre del muñón rectal. La biopsia informó segmento de intestino delgado con hallazgos compatibles con enteropatía isquémica aguda. Evolucionó con flujos de $4.500 \mathrm{cc} /$ día por yeyunostomía y desnutrición calórico-proteica. Manejada inicialmente con NPTC, en el día 12 post operatorio se inició alimentación por sonda nasoenteral y en el día 50 se logró alimentación oral exclusiva. Al alta toleró bien régimen oral con suplementos nutricionales.

Hasta junio 2008 presentó 17 hospitalizaciones, 4 por cuadros de íleo mecánico, todos resueltos sin necesidad de cirugía, y 13 por episodios de deshidratación moderada secundaria a altos débitos de la ostomía, los que se manejaron con reposición hidroelectrolítica y ajuste de fármacos para disminuir flujo por yeyunostomía logrando volumen de deposiciones de 2.000-3.000 cc/día.

El 26/06/08 ingresó nuevamente por cuadro de deshidratación grave. Se constató creatininemia de 4,39 mg/dl, objetivándose clearence de creatinina de $30 \mathrm{ml} / \mathrm{min}$. Dado el deterioro de la función renal y compromiso nutricional de predominio calórico con IMC de $14,2 \mathrm{~kg} / \mathrm{m}^{2}$ y albúmina de ingreso de $3,1 \mathrm{gr} / \mathrm{dl}$, se planteó la realización de un procedimiento de elongación intestinal y reconstitución del tránsito para mejorar la condición clínica y calidad de vida de la paciente.

El estudio pre-operatorio incluyó una rectoscopia rígida que evidenció un muñón rectal de $12 \mathrm{~cm}$. El tránsito intestinal demostró $140 \mathrm{~cm}$ de intestino delgado sin estrecheces y leve dilatación de asas. Por sospecha en la tomografía computada de compromiso de la arteria mesentérica superior (AMS), se realizó ecografía Doppler que mostró estenosis significativa (70\%) de la AMS, que se resolvió mediante angioplastía y colocación de endoprótesis. Con aporte de régimen hipercalóricohiperproteico y NPTC se logró optimización de parámetros nutricionales (albúmina: $3,8 \mathrm{mg} / \mathrm{dl}$ ). 

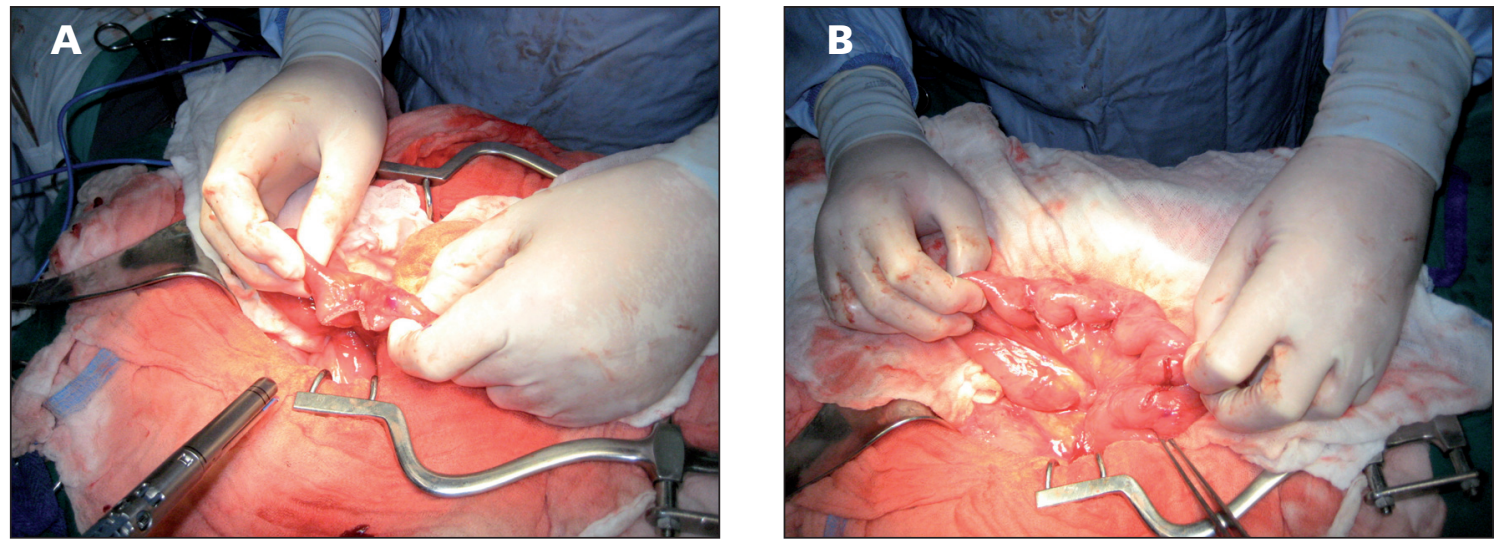

Figura 2. A: Sección transversa. B: Intestino en forma de zig-zag.

Cinco semanas luego de la colocación de la endoprótesis se decidió operar a la paciente. En el intraoperatorio se midió intestino remanente confirmando una longitud de $140 \mathrm{~cm}$. Se desmanteló la yeyunostomía y se practicó una anastomosis mecánica término-terminal yeyunorectal. Luego se hicieron 15 secciones transversas parciales con cargas lineales de 3,5 $\mathrm{mm}$ para seccionar la mitad del lumen del intestino en forma de zig-zag (Figura 2) según lo descrito por Kim9. La longitud final del intestino delgado fue de $180 \mathrm{~cm}$, lo que sumado al muñón rectal da una longitud de 190 $\mathrm{cm}$ hasta el ano.

Dentro de los eventos del postoperatorio destacó la formación de colecciones hemáticas intraabdominales, sin evidencias de filtración. Por persistencia de episodios febriles, se realizó ecocardiograma que evidenció presencia de trombo séptico en aurícula derecha por lo que se inició tratamiento para endocarditis bacteriana.

La paciente evolucionó afebril, con aumento progresivo del aporte por vía oral. El día 34 del post operatorio se suspendió la NPTC, logrando balance hidroelectrolitico y nutricional adecuado exclusivamente por vía oral. El estudio de tránsito intestinal a los 98 días post cirugía no demostró evidencias de las enterotomías.

Actualmente en control ambulatorio, en tratamiento con loperamida y suplementos nutricionales, logrando adecuado balance hidroelectrolítico, aumento de peso y con una frecuencia de deposiciones de 5 veces por día con escurrimiento escaso.

\section{Discusión}

Diversas técnicas quirúrgicas han sido desarrolladas para el manejo del SIC, las que pueden agruparse en procedimientos que aumentan el tiempo de transito intestinal, técnicas que evitan el sobrecrecimiento bacteriano, procedimientos de elongación intestinal y finalmente el $\mathrm{TI}^{10-12}$. En la actualidad, las técnicas de alargamiento de la longitud del intestino remanente son las más utilizadas y de las que se posee mayor evidencia teórica y clínica, entre las que destacan la técnica de Bianchi y la técnica de STEP.

La técnica de Bianchi ${ }^{8}$ consiste en la división longitudinal de intestino en 2 mitades de igual diámetro las cuales preservan su vascularización y que posteriormente se anastomosan una tras la otra de forma isoperistáltica, con lo que se dobla la longitud inicial del intestino. Se recomienda la utilización de este procedimiento en pacientes con intestino de diámetro mayor a $3 \mathrm{~cm}$ y con una longitud de intestino remanente de a lo menos $40 \mathrm{~cm}^{13,14}$.

La técnica de STEP se basa en que el aporte sanguíneo intestinal proviene del borde mesentérico y lo atraviesa de forma transversal y perpendicular a su eje longitudinal ${ }^{9}$, por lo que la sección transversal del intestino en forma de zig-zag utilizada para lograr el aumento en la longitud no afecta la irrigación ni funcionalidad del intestino remanente. La nueva longitud intestinal dependerá del grado de dilatación previo a la cirugía y del tamaño del lumen del intestino creado, lo que se relaciona a 
su vez con la longitud de cada corte y el número de cortes realizados.

Este procedimiento fue descrito por Kim en $2003^{9,15}$. En modelos animales de SIC, la realización de STEP ha demostrado un incremento en la longitud del intestino residual en aproximadamente $50 \%{ }^{9,16}$, lo que asocia a un aumento en la captación de hidratos de carbonos medidos por la absorción intestinal de D-Xilosa y a un aumento en la capacidad de absorción de grasas evaluado por los niveles plasmáticos de triglicéridos y vitamina D. Se describió a su vez una elevación en los niveles séricos de citrulina, secundario a un incremento de la masa de la mucosa intestinal. Los resultados prometedores de esta técnica permitieron a los autores concluir que dicho procedimiento podría ser indicado a pacientes seleccionados con SIC refractario a tratamiento médico ${ }^{16}$.

Recientemente se presentaron los resultados del primer registro internacional de esta técnica ${ }^{17}$. Se describen como resultados postoperatorios un aumento de la longitud intestinal, desde un promedio de $68 \mathrm{~cm}$ a $115 \mathrm{~cm}$ post-STEP y un incremento significativo en la tolerancia a la nutrición enteral desde $31 \%$ a $67 \%$ de calorías aportadas en paciente con dependencia previa de NPTC. Diez pacientes se independizaron completamente del uso de NPTC. A largo plazo sólo 3 pacientes requirieron TI.

La necrosis intestinal que dio origen al SIC de esta paciente sin duda tuvo relación con su colectomía, procedimiento en el que se habría comprometido la AMS, quedando esta última con una estrechez significativa aunque aparentemente sin impacto clínico para el segmento intestinal remanente. Entre los factores que contribuyen al estado actual de la paciente se encuentran la resección masiva de intestino delgado, la falta de continuidad del tracto digestivo y la ausencia de válvula ileocecal. Así, el tratamiento quirúrgico de la paciente incluyó la corrección de la estenosis de la AMS, el reestablecimiento de la continuidad del tubo digestivo mediante la anastomosis yeyunorectal, y además se realizó un STEP con el fin de disminuir el impacto del intestino corto. Se optó por un STEP ya que no requiere anastomosis intestinales y presentaría un menor riesgo de lesión de los vasos mesentéricos que el método de Bianchi. Si bien no es posible atribuir sólo al STEP la mejoría clínica de la paciente, sino que a la combinación de los procedimientos realizados, la evidencia presentada en la literatura permite suponer que la elongación del intestino es clave para lograr estos resultados.

Hasta nuestro conocimiento, este es el primer caso descrito en nuestro país de una paciente con SIC sometida a STEP, la cual se encuentra actualmente en buenas condiciones y con una mejoría significativa en su calidad de vida. Existe evidencia teórica y clínica que apoya la utilización del STEP como una técnica quirúrgica segura y eficaz para el tratamiento de pacientes con SIC, pudiendo ser una alternativa al TI en pacientes seleccionados.

\section{Referencias}

1. Ballesteros M, Vidal A. Short bowel syndrome: definition, causes, intestinal adaptation and bacterial overgrowth. Nutr Hosp 2007; 22 Suppl 2: 74-85.

2. Cavicchi M, Beau P, Crenn P, Degott C, Messing B. Prevalence of liver disease and contributing factors in patients receiving home parenteral nutrition for permanent intestinal failure. Ann Intern Med 2000; 132: 525-32.

3. Mahgoub L, Puntis J. Long-term parenteral nutrition. Current Paediatrics 2006; 16: 298-304.

4. Diamanti A, Basso Ms, Castro M, Calce A, Pietrobattista A, Gambarara M. Prevalence of life-threatening complications in pediatric patients affected by intestinal failure. Transplant Proc 2007; 39: 1632-3.

5. Kaufman SS, Atkinson JB, Bianchi A, Goulet OJ, Grant $\mathrm{D}$, Langnas AN, et al. Indications for pediatric intestinal transplantation: a position paper of the American Society of Transplantation. Pediatr Transplant 2001; 5: 80-7.

6. Atalay F, Ozcay N, Gundogdu H, Orug T, Gungor A, Akoglu M. Evaluation of the outcomes of short bowel síndrome and indications for intestinal transplantation. Transplant Proc 2003; 35: 3054-6.

7. Grant D, Abu-Elmagd K, Reyes J, Tzakis A, Langnas A, Fishbein T, et al. 2003 report of the intestine transplant registry: a new era has dawned. Ann Surg 2005; 241: 607-13.

8. Bianchi A. Intestinal loop lenghtening. A technique for increasing small intestinal lenght. J Pediatr Surg 1980; 15: 145-51.

9. Kim H, Fauza D, Garza J, Oh Jt, Nurko S, Jaksic T. Serial transverse enteroplasty (STEP): a novel bowel lengtening procedure. J Pediatr Surg 2003; 38: 425-9.

10. Bueno J. Surgical strategies in short bowel síndrome. Nutr Hosp 2007; 22 Suppl 2: 103-12.

11. Wales P. Surgical therapy for short bowel syndrome. 
Enteroplastía transversa seriada en el tratamiento del síndrome de intestino corto - F. Bellolio R. et al

Pediatr Surg Int 2004; 20: 647-57.

12. Barksdale E, Stanford A. The surgical management of short bowel syndrome. Curr Gastroenterol Rep 2002; 4: 229-37.

13. Bianchi A. Longitudinal intestinal lengthening and tailoring: results in 20 children. J R Soc Med 1997; 90: 429-32.

14. Sudan D, Thompson J, Botha J, Grant W, Antonson D, Raynor S, et al. Comparison of intestinal lengthening procedures for patients with short bowel syndrome. Ann Surg 2007; 246: 593-601.
15. Kim Hb, Lee Pw, Garza J, Duggan C, Fauza D, Jaksic T. Serial transverse enteroplasty for short bowel syndrome: a case report. J Pediatr Surg 2003; 38: 881-5.

16. Chang RW, Javid PJ, Oh JT, Andreoli S, Kim HB, Fauza $\mathrm{D}$, et al. Serial transverse enteroplasty enhances intestinal function in a model of short bowel syndrome. Ann Surg 2006; 243: 223-8.

17. Modi B, Javid P, Jaksic T, Piper H, Langer M, Duggan C, et al. First report of the international serial transverse enteroplasty data registry: indications, efficacy, and complications. J Am Coll Surg 2007; 204: 365-71. 